\title{
Chrysin Attenuates the NLRP3 Inflammasome Cascade to Reduce Synovitis and Pain in KOA Rats
}

This article was published in the following Dove Press journal: Drug Design, Development and Therapy

\author{
Taiyang Liao $\mathbb{1}^{1-3, *}$ \\ Liang Ding ${ }^{1,2, *}$ \\ Peng $\mathrm{Wu}^{1-3, *}$ \\ Li Zhang ${ }^{1-3}$ \\ Xiaochen $\mathrm{Li}^{1-3}$ \\ Bo $\mathrm{Xu}^{\mathrm{I}-3}$ \\ Haosheng Zhang ${ }^{1-3}$ \\ Zhenyuan $\mathrm{Ma}^{\mathrm{I}-3}$ \\ Yancheng Xiao ${ }^{1-3}$ \\ Peimin Wang ${ }^{1,2}$
}

'Department of Orthopedics, The Affiliated Hospital of Nanjing University of Chinese Medicine, Nanjing, Jiangsu 210029 , People's Republic of China; 2Jiangsu Province Hospital of Chinese Medicine, Nanjing, Jiangsu 210029,

People's Republic of China; ${ }^{3}$ Key Laboratory for Metabolic Diseases in Chinese Medicine, First College of Clinical Medicine, Nanjing University of Chinese Medicine, Nanjing, Jiangsu 210029, People's Republic of China

*These authors contributed equally to this work
Correspondence: Peimin Wang

Department of Orthopedics, The Affiliated Hospital of Nanjing University of Chinese

Medicine, No. 155 Hanzhong Road, Qinhuai

District, Nanjing, Jiangsu 210029, People's

Republic of China

Tel +86 I5279703634

Email drwpm@163.com
Purpose: Our recent reports have revealed that inhibiting NLRP3 activation reduces synovial inflammation and fibrosis in knee osteoarthritis (KOA). Synovial inflammation is involved the entire process of KOA and promotes the progression of KOA. Natural flavonoid Chrysin from Scutellariae Radix, a traditional Chinese medicine, exhibits multifarious biological activities and potentially has protective activity against osteoarthritis. However, the mechanism of Chrysin in the treatment of synovial inflammation remains elusive. The purpose of our research was to explore the anti-inflammatory effects of Chrysin on KOA, which was induced by monoiodoacetic acid (MIA) in rats by targeting the NLRP3 inflammasome in the hopes of identifying an effective drug to treat KOA.

Methods: The MIA-induced KOA model was used to evaluate the cold pain threshold and paw withdrawal threshold (PWT) of joints after MIA $(40 \mathrm{mg} / \mathrm{mL})$ injection into the knee joints. Microscopically, we used LPS (5 ug/mL) and ATP (4 mmol/L) to stimulate fibroblast-like synovial cells (FLSs) to explore the underlying mechanisms and effects of Chrysin. Two staining methods, H\&E and Sirius Red, were applied to assess histopathological changes in synovial membranes. Cellular signal transduction was determined by qRT-PCR and WB. Cytokine expression (inflammatory cytokines and pain-related cytokines) was detected by ELISA. The degree of chronic inflammatory pain was evaluated by c-Fos immunofluorescence.

Results: The results showed that Chrysin not only attenuated synovial inflammation but also reduced the secretion of pain-related factors and increased the PWT and cold pain threshold in rats. Chrysin also inhibited NLRP3 inflammasome activation and increased IL-1 $\beta$ levels to alleviate the synovitis.

Conclusion: Chrysin can relieve knee synovial inflammation and improve pain behavior in KOA rats, which may be related to the ability of Chrysin to inhibit NLRP3 inflammasome activation. Therefore, Chrysin may be developed as a new drug for the treatment of KOA.

Keywords: KOA, Chrysin, synovitis, pain, NLRP3 inflammasome

\section{Introduction}

KOA is a chronic inflammatory condition with persistent painful arthritis that can lead to decompensation and severe disability and represents a significant strain on the health system and social economy. In addition, rates continue to increase due to many causes, such as obesity, femaleness, aging, diet, and joint damage. ${ }^{1}$ Incomplete statistics show that 250 million people worldwide are currently affected. ${ }^{2}$ Treatments, including pain relief or joint replacement, are not curative, but neither method exhibits potentially harmful side effects. Synovitis is a typical 
pathological change of osteoarthritis, and no specific drugs effectively treat osteoarthritis. ${ }^{3,4}$ Although the cause of KOA remains unclear, inflammation exhibits an important correlation in the pathogenesis of osteoarthritis. ${ }^{5}$ Joint inflammation directly leads to the onset of pain in KOA, exacerbates cartilage damage, and may lead to continued sensitization of pain, which eventually evolves into chronic pain. ${ }^{6,7}$

The NLRP3 inflammasome is involved in the pathogenesis of many forms of arthritis. ${ }^{8}$ NLRP3 inflammasomes are assembled by the formation of a macromolecular complex via the recruitment of ASC and the serine protease caspase-1. ${ }^{9,10}$ Subsequently, activated caspase-1 further leads to the release of two proinflammatory cytokines, IL-1 $\beta$ and IL-18, which leads to cartilage degeneration and synovial membrane inflammation. ${ }^{11,12}$

Chrysin is a natural flavonoid that is present in many herbal plant extracts, honey and propolis. ${ }^{13,14}$ It is also the main ingredient of Scutellariae Radix, ${ }^{15}$ which is a kind of traditional Chinese medicine that is derived from natural products. It is cultivated in many areas of China and has been used to treat many diseases since ancient times. Recent papers have demonstrated that Chrysin exhibits a variety of bioactivities, including immunomodulatory, ${ }^{16}$ anti-inflammatory, ${ }^{17,18}$ anti-oxidative stress, ${ }^{19,20}$ and neuroprotective effects. ${ }^{21}$ However, previous studies on Chrysin mostly focused on its effects on cartilage tissue for the treatment of cartilage dysfunction. ${ }^{17}$ Little is known about whether the treatment of Chrysin improves KOA inflammation via the synovium and FLSs. Furthermore, whether Chrysin affects FLSs by inhibiting NLRP3 inflammasome activation and Interleukin-1 $\beta$ secretion in KOA remains unknown. At this stage, our basic experiment investigated the potential therapeutic role of Chrysin in KOA, which may represent a new drug for clinical treatment.

\section{Materials and Methods}

\section{Reagents}

Chrysin ( $>98 \%$ purity) was obtained from Yuanye (Shanghai, China). Chrysin was dissolved in dimethylsulfoxide ( $<1 \%$ DMSO) and freshly diluted in culture media for all in vitro experiments. Monoiodoacetic acid, lipopolysaccharide (LPS), type I collagenase and DMSO were all obtained from Sigma-Aldrich (Sigma, St.Louis, MO, USA). TRIzol, Dulbecco's Modified Eagle Culture Medium (DMEM), Penicillin-Streptomycin mixture, fetal bovine serum (FBS) were purchased from Gibco (Rockville, USA). The primary antibodies for GAPDH, NLRP3, Caspase-1, Interleukin-18, Interleukin-1 $\beta$ and ASC were all purchased from Abcam (Cambridge, UK). Antibodies for c-Fos were purchased from Servicebio (Wuhan, China). Picro Sirius Red Stain kit and Goat anti-rabbit IgG H\&L (HRP) were also supplied by Abcam (Cambridge, UK). In addition, $5 \times$ HiScript II qRt SuperMix and $2 \times$ ChamQ SYBR qPCR MsterMix (Low ROX Premixed) were obtained from Vazyme (Nanjing, China). The primers were supplied by Sangon Biotech (Shanghai, China). Caspase-1 Activity Assay Kit and Cell-Counting Kit-8 were obtained from Solarbio (Beijing, China). Enzyme-linked immunosorbent assays (ELISA) kits for Interleukin- $1 \beta$ and Interleukin-18 were supplied by Invitrogen (Life Technologies Corp., California, USA). ELISA kits for CGRP and SP were supplied by Jin Yibai (Nanjing, China).

\section{In vivo Animal Experimental Design}

The rats' experiments were ethically conducted and approved by the Institutional Animal Care and Use Committee of Nanjing University of Chinese Medicine (Application number: 201905A002) and were conducted in accordance with the National Institutes of Health Guide for the Care and Use of Laboratory animals. Eight-week-old male Sprague Dawley (SD) rats (Nanjing Qinglong-shan Animal Farm, License number: SCXK-SU- 2017-0001) weighing $150-200 \mathrm{~g}$ were randomly divided into 3 groups: Normal group ( $\mathrm{n}=10)$, MIA group $(\mathrm{n}=10)$, and MIA + Chrysin group $(\mathrm{n}=10)$. Rats were housed in an SPFgrade environment with controlled temperature and humidity and free of specific pathogens. Rat food and water were available ad libitum. After one week of feeding, these rats were grouped and numbered. In the first 14 days, ten rats in the normal group were injected with $50 \mathrm{ul}$ sterilized physiologic saline, and the KOA model was constructed by MIA as previously mentioned, using both knees. ${ }^{22}$ From the 14th day, Chrysin (oscillated dissolved in $0.5 \%$ sodium carboxymethylcellulose (CMC) overnight) was administered by intragastric administration. The normal group and the MIA group were administered $0.5 \% \mathrm{CMC}$ as the control, and each rat in the MIA+Chrysin group was treated with a dose of $10 \mathrm{mg} / \mathrm{kg}$ Chrysin. ${ }^{23}$ The above three groups were given gavaged once a daily for 14 days. At day 28, we sacrificed the rats by intraperitoneal injection of $3 \%$ pentobarbital sodium $(100 \mathrm{mg} / \mathrm{kg})$, and all animals were sacrificed to harvest the synovial tissue, cartilage, dorsal root ganglion and plasma. 


\section{Measurements of the PWT and Cold Pain Threshold}

The PWT was measured at $0,7,14,21$, and 28 days. The modeling period was $0-14$ days, and the administration period was 14-28 days. PWT measurements were performed as described previously. ${ }^{24}$ The simple procedure is described as follows: the calm rats were placed in a plexiglass cage (Institute of Biomedical Engineering, Chinese Academy of Medical Sciences, BME404). The electron needle stimulates the sole of the right hind foot, and its stimulation intensity gradually increases. The computer recorded the values if the rat exhibits rapid reactions, such as clawing, lifting and licking its feet. This process was repeated thrice with a 20-minute interval between each test to obtain an average value.

Rats were placed sequentially on a temperature-adjustable cold plate $\left(0 \pm 1^{\circ} \mathrm{C}, 35,150,001\right.$, Ugo Basil SLR, Italy), and calm rats were also covered with an organic cylinder. ${ }^{25}$ At this point, the operator immediately started timing. As time passed, the rats would quickly withdraw their paw, lick its paw, and stamp or jump because they could not tolerate the low temperature, and the operator would immediately pause the timer and recorded the duration. Paw retraction due to the rat's own activity halfway through the experiment was not recorded. During the five minutes between each test, the cold plate was cleaned. A total of three measurements were obtained, and the average was calculated.

\section{Histological Analysis}

After the administration of Chrysin, we collected all the knee joints of the rats and fixed a part of the knee joints with paraformaldehyde overnight (4\%) followed by decalcification (10\% EDTA), paraffin embedding and sagittal sections (cut at approximately $5 \mu \mathrm{m}$ ). The degree of synovitis was assessed by $H \& E$ staining. The conventional procedures for Sirius red staining were similar to those for H\&E staining. After conventional treatment, the sections were dipped into the staining solution (including Picric Acid and Sirius Red) at room temperature for just one hour and rinsed gently under running water for approximately 10 minutes. The degree of synovitis histological observation was performed using a DMI3000B microscope (Leica, Germany) system.

\section{The Method of Obtaining DRG}

The spinal column was separated from the dorsal side of the rat, and then washed with prechilled PBS solution to remove contaminated blood. The spinal column was trimmed on ice. Under the Leica S6 D stereomicroscope, the spine was divided into two halves along the midline of the abdomen of the spine using ophthalmic scissors. The spinal cord was removed. The DRG was exposed, and the DRG was carefully removed with forceps. The connected nerve fibers were cut off and obtain the tissues of DRG.

\section{Immunofluorescence Assay}

The DRG samples were dissected and fixed with $4 \%$ paraformaldehyde. Then, DRG were placed in xylene for 15 minutes and anhydrous ethanol for 5 minutes. Next, BSA serum was added, and the sample was incubated for 30 minutes. Subsequently, the slices were incubated in primary antibody overnight in a wet box for $4^{\circ} \mathrm{C}$ followed by incubation for 50 minutes at $4^{\circ} \mathrm{C}$ with secondary antibodies. Finally, the DRG c-Fos-immunostained sections were assessed using a confocal scanning microscope (Zeiss LSM 700).

\section{In vitro Preparation and Stimulation of Primary Rat Fibroblast-Like Synoviocytes}

First, synovial tissue was extracted, washed twice with PBS, cut into $1-2 \mathrm{~mm}^{2}$ pieces using scissors, and homogenized in basic medium. Then, $5 \mathrm{~mL}$ of $0.2 \%$ type I collagenase was added, and samples were incubated for $2 \mathrm{~h}$ at $37^{\circ} \mathrm{C}$ in a cell culture chamber containing $5 \% \mathrm{CO}_{2}$. Collagenase dissociated FLSs from synovial tissue. Then, FLSs were subject to filtration, centrifugation, precipitation, and resuspension. Then, $5-10 \mathrm{~mL}$ of complete culture medium containing $10 \%$ FBS and $1 \%$ penicillin-streptomycin solution were added to the culture flask. The medium should be replaced every other day and then every two days. After approximately one week, cells grew along the wall. To ensure good cellular activity, 3rd to 6th generation FLSs were used in the experiment. Cell identification was performed as described in our previous research. ${ }^{26}$

We divided the experiment into three groups: Normal group, LPS group and LPS + Chrysin group. The treatment used the LPS + Chrysin group was summarized as follows. Synovial cells were digested from the culture flask and inoculated in a 6-well plate for $24 \mathrm{~h}$. On the next day, the complete culture medium was changed to serum-free medium. Finally, FLSs were treated with LPS $(5 \mu \mathrm{g} / \mathrm{mL})$ in PBS for $6 \mathrm{~h}$, and ATP $(4 \mathrm{mmol} / \mathrm{L}$ ) was added for $2 \mathrm{~h}$ to stimulate the inflammatory response and activate the NLRP3 inflammasome. Then, the culture medium was changed, and Chrysin $(5 \mathrm{ug} / \mathrm{mL})$ was added for $24 \mathrm{~h}$ or $48 \mathrm{~h}$ in subsequent experiments. 


\section{Cell Cytotoxicity Assays}

The cytotoxic effects of Chrysin on FLSs were investigated using CCK-8 assays. Briefly, 8000 FLSs per well were cultured for $24 \mathrm{~h}$ in a 96-well plate and then treated with DMSO alone or Chrysin $(1,2,2.5,5,10,20$, or $40 \mathrm{ug} / \mathrm{mL})$ for 24 or $48 \mathrm{~h}$, respectively. Then, $10 \mu \mathrm{L}$ CCK-8 solution was added to each well, and the plates were placed in the incubator for 4 hours. The absorbance of each well was read on a microplate spectrophotometer (PE EnSpire) at $450 \mathrm{~nm}$.

\section{Caspase-I Activity Detection}

These steps were performed per the producer's instructions. First, FLSs were lysed in $100 \mu \mathrm{L}$ lysis buffer at $0^{\circ} \mathrm{C}$ for 15 minutes. The Bradford method was applied to detect supernatant protein concentrations $(1-3 \mu \mathrm{g} / \mu \mathrm{L})$. Next, the optical density (OD) value was measured at $450 \mathrm{~nm}$, and caspase-1 activity was obtained by generating a PNA standard curve.

\section{Western Blot}

The required total protein was isolated from synovial tissues and cultured FLSs. These samples were lysed for one hour with RIPA lysate containing $0.1 \%$ PMSF (phenylmethanesul fonylfluoride) in a box filled with ice. The lysates were spun in a centrifuge for 20 minutes at $4^{\circ} \mathrm{C}$ and $12,000 \mathrm{rpm}$, and clear supernatant was collected. A 10-ul aliquot of the supernatant was used to prepare the standard curve with the bicinchoninic acid protein detection kit (Beyotime, Nanjing, China). Specifically, the absorption value at $562 \mathrm{~nm}$ was used to determine the protein concentration. The remaining protein was added to $100 \mu \mathrm{L}$ SDS-PAGE buffer (5X), and the protein was boiled at $99^{\circ} \mathrm{C}$ for 10 minutes. Then, an SDS gel was prepared and SDS-PAGE was electrophoresed for 90 minutes to separate the protein bands. It took approximately 1 hour to transfer the protein from the gel to the PVDF membrane. Then, the membrane was blocked with 5\% BSA in TBST buffer for 1 hour at room temperature and then washed thrice with TBST for $10 \mathrm{~min}-$ utes each time. The membrane was incubated with the first antibody (1:1000) overnight in the cold storage. On the next day, the membrane was incubated in the second antibody (1:3000) for $1 \mathrm{~h}$. Then, the PVDF membrane was washed thrice with TBST and exposed to $200 \mu \mathrm{L}$ ECL solution. Finally, the overall gray value of protein bands was analyzed using ImageJ 7.04.

\section{Quantitative Real-Time Polymerase Chain Reaction (qRT-PCR)}

First, total RNA was extracted from synovial tissue and FLS using Trizol solution, and the concentration and purity of RNA were measured using a spectrophotometer. The ratio of the OD value at 260/280 $\mathrm{nm}$ should be between 1.8 and 2.0. Next, a total of $20 \mu \mathrm{L}$ of the reaction mixture (including $4 \mu \mathrm{L}$ $5 \times$ HiScript II qRT Super Mix) was subject to reverse transcription of total RNA into stable CDNA under the following conditions: $50^{\circ} \mathrm{C}(15 \mathrm{~min})$ and $85^{\circ} \mathrm{C}(5 \mathrm{~s})$. The primer sequences were shown in Table 1. PCR reactions (per well: $0.4 \mu \mathrm{L}$ each of forward and reverse primers, $10 \mu \mathrm{L} 2 \times$ ChamQ SYBR qPCR Master Mix (Low ROX Premixed), $1 \mu \mathrm{L}$ CDNA, $8.2 \mu \mathrm{L}$ ddH2O; 3 replicate wells) were performed using ABI 7500 qRT-PCR system (Applied Biosystems, USA). The following reaction conditions were employed: the first stage (pre-denaturation), $95^{\circ} \mathrm{C}$ for $30 \mathrm{~s}$; the second stage (denaturation), $95^{\circ} \mathrm{C}$ for $10 \mathrm{~s}$ and $60^{\circ} \mathrm{C}$ for $30 \mathrm{~s}$; the third stage (melting curve), $95^{\circ} \mathrm{C}$ for $15 \mathrm{~s}, 60^{\circ} \mathrm{C}$ for $60 \mathrm{~s}$, and $95^{\circ} \mathrm{C}$ for $15 \mathrm{~s}$. GAPDH was used as a housekeeping gene, and independent experiments were repeated at least thrice. Expression levels were calculated using the $2^{-\Delta \Delta}$ CT data analysis method.

\section{ELISA}

We prepared cell supernatants and rat serum in advance for the experiment, allowing all reagents to reach room temperature before use. Then, the ELISA procedure was performed according to the manufacturer's instructions. Optical density (OD) values were measured at $450 \mathrm{~nm}$, and standard curves were plotted to calculate the levels of IL-1 $\beta$, IL-18, CGRP and SP.

Table I Primers

\begin{tabular}{|l|l|l|}
\hline Gene & Forward Primer (5'-3') & Reverse Primer (5'-3') \\
\hline NLRP3 & GAGCTGGACCTCAGTGACAATGC & ACCAATGCGAGATCCTGACAACAC \\
Caspase-I & ATGGCCGACAAGGTCCTGAGG & GTGACATGATCGCACAGGTCTCG \\
ASC & AGAGTCTGGAGCTGTGGCTACTG & ATGAGTGCTTGCCTGTGTTGGTC \\
$I L-I \beta$ & ACAGCAGCATCTCGACAAGAGC & CCACGGGCAAGACATAGGTAGC \\
$I L-I 8$ & TCTGTAGCTCCATGCTTTCCG & GATCCTGGAGGTTGCAGAAGA \\
\hline
\end{tabular}




\section{Statistical Analysis}

All experiments were performed independently at least thrice, and data were expressed as mean \pm standard deviation (SD). Statistical analyses were performed with GraphPad Prism 7.04 Software (San Diego, CA, USA) and statistical significance was indicated by $\mathrm{P}$-value $<0.05$. Comparisons between the three groups were conducted Student's $t$-test, one-way ANOVA or two-way ANOVA.

\section{Results}

\section{Chrysin Reduced MIA-Induced Synovitis in vivo}

Chrysin is a natural flavonoid that is extracted from Scutellariae Radix (Figure 1A). First, rats in our experiment were divided into three groups. MIA $(40 \mathrm{mg} / \mathrm{mL})$ was used for modeling and intragastric administration, and a number of time intervals were used for measurements and sampling (Figure 1B). MIA-induced synovitis causes a series of pathological changes in synovial tissues. Therefore, we performed H\&E and Sirius Red Staining of the synovial sections to evaluate the therapeutic effect of Chrysin on synovitis. For H\&E staining of MIA group samples, "A arrow" indicates inflammatory cell infiltration, "B arrow" indicates thickening of synovial lining, and " $\mathrm{C}$ arrow" indicates synovial fibrosis. ${ }^{27}$ However, in MIA+Chrysin group, "A arrow" indicates had less inflammatory cell infiltration, "B arrow" and " $\mathrm{C}$ arrow" indicate reduced hyperplasia and fibrosis, respectively. Moreover, type I collagen deposition was increased in the MIA group, while this tendency was reduced in the MIA + Chrysin group as assessed by Sirius Red staining (Figure 1C). Furthermore, through quantitative analysis of protein and genes, we found that compared with the normal group, IL-1 $\beta$ and IL-18 expression levels in synovial tissues of the MIA group exhibited a significant upward trend. This increasing change was significantly suppressed in the MIA + Chrysin group (Figure 1DF). Finally, we detected the secretion of inflammatory factors IL-1 $\beta$ and IL-18 in rat serum, and secretion was also inhibited by Chrysin (Figure 1G).

\section{Chrysin Suppressed MIA-Induced NLRP3 Inflammasome Activation in vivo}

Next, we synthetically analyzed the effects of Chrysin on NLRP3, caspase-1 and ASC protein and mRNA expression levels in rat synovial tissue. The NLRP3 inflammasome is closely related to the pathogenesis of KOA, and NLRP3 inflammasome activation results in severe synovitis as previously described. Western blot confirmed that Chrysin significantly reduces increases in NLRP3, caspase-1 and ASC at the protein level (Figure 2AB). MIA modeling resulted in increased NLRP3, caspase-1 and ASC mRNA expression in synovial tissue compared with the normal group, but Chrysin reverses this effect (Figure 2C).

\section{Chrysin Alleviated Pain and Increased the Cold Pain Threshold (s) and PWT (g) in vivo}

Subsequently, c-Fos expression in DRG was analyzed by immunofluorescence. After MIA injection, c-Fos protein expression in DRG was rapidly increased in neurons after harmful stimuli, such as inflammation and stress response. This increase was suppressed by Chrysin (Figure 3AB). We also observed that MIA-induced cold pain threshold and PWT, which are often used to assess peripheral pain sensitivity, were increased in KOA rats after treatment with Chrysin (Figure 3CD). Finally, we examined the effect of chrysin on CGRP and SP expression in rat serum. The results showed that Chrysin treatment dramatically reduced CGRP and SP expression (Figure 3E).

\section{Chrysin Reduced Inflammatory Factors in LPS-Induced FLSs in vitro}

To confirm whether Chrysin could inhibit NLRP3 inflammasome activation, FLSs were exposed to $5 \mathrm{ug} / \mathrm{mL}$ of LPS for $6 \mathrm{~h}$. Then, ATP $(4 \mathrm{mmol} / \mathrm{L})$ was added for $2 \mathrm{~h}$, and Chrysin was added for $24 \mathrm{~h}$ for subsequent experiments. The CCK-8 assay was initially performed to investigate the cytotoxicity of different Chrysin concentrations (1, 2, 2.5, 5, 10,20 , and $40 \mathrm{ug} / \mathrm{mL}$ ) in FLSs. No significant difference in cell viability was noted with Chrysin $(0-5 \mathrm{ug} / \mathrm{mL})$ treatment compared with controls, indicating that Chrysin does not induce cytotoxic effects in FLSs (Figure 4A). However, cytotoxic effects were observed with $10 \mathrm{ug} / \mathrm{mL}$ Chrysin. Therefore, Chrysin at $5 \mathrm{ug} / \mathrm{mL}$ was used in subsequent experiments. To determine the influence of Chrysin on the cytokine expression in the rat MIA model of osteoarthritis, IL-1 $\beta$ and IL-18 levels in FLSs were measured by WB (Figure 4BC) and qRT-PCR (Figure 4D). Compared with the Normal group, protein and mRNA expression of all detected cytokines in the LPS group were significantly upregulated $(\mathrm{P}<0.05)$. In contrast, the levels of all detected cytokines exhibited remarkable downregulation in the Chrysin-administered group $(\mathrm{P}<0.05)$. Moreover, ELISA results showed that the expression of the proinflammatory 
A<smiles>O=c1cc(-c2ccccc2)oc2cc(O)cc(O)c12</smiles>

Chemical structure of Chrysin Molecular Formula: $\mathrm{C}_{15} \mathrm{H}_{10} \mathrm{O}_{4}$ Molecular Weight: $254.24 \mathrm{~g} / \mathrm{mol}$

C

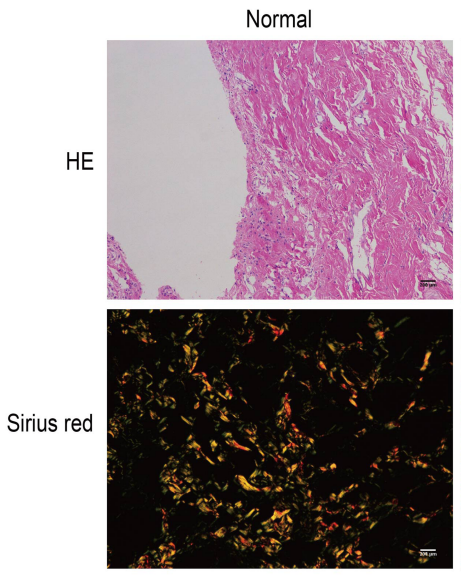

D

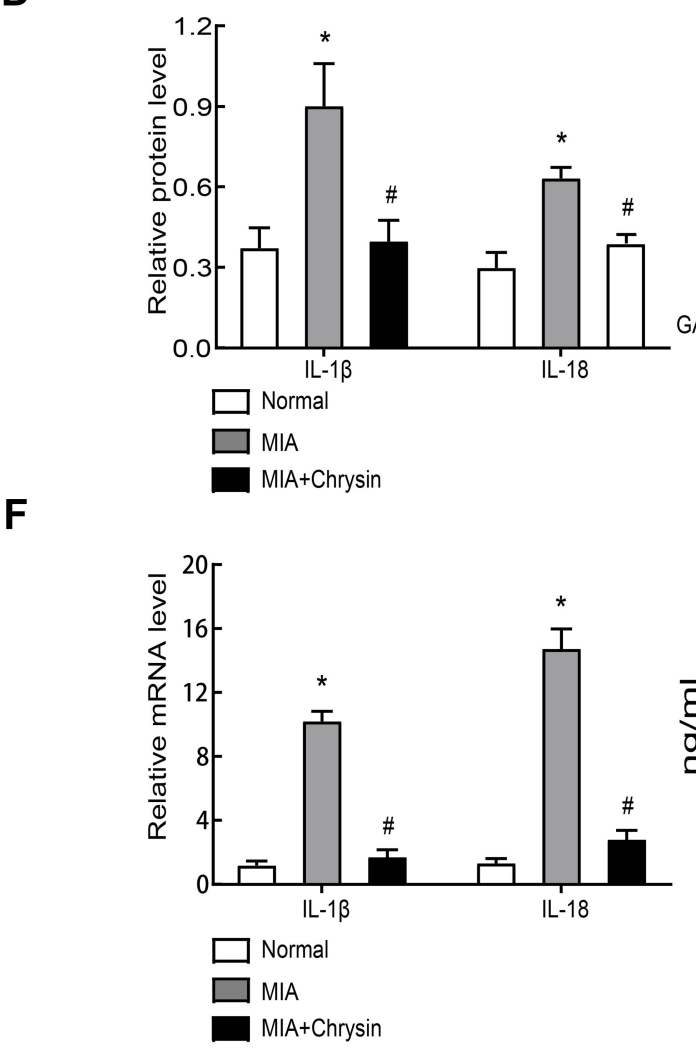

MIA
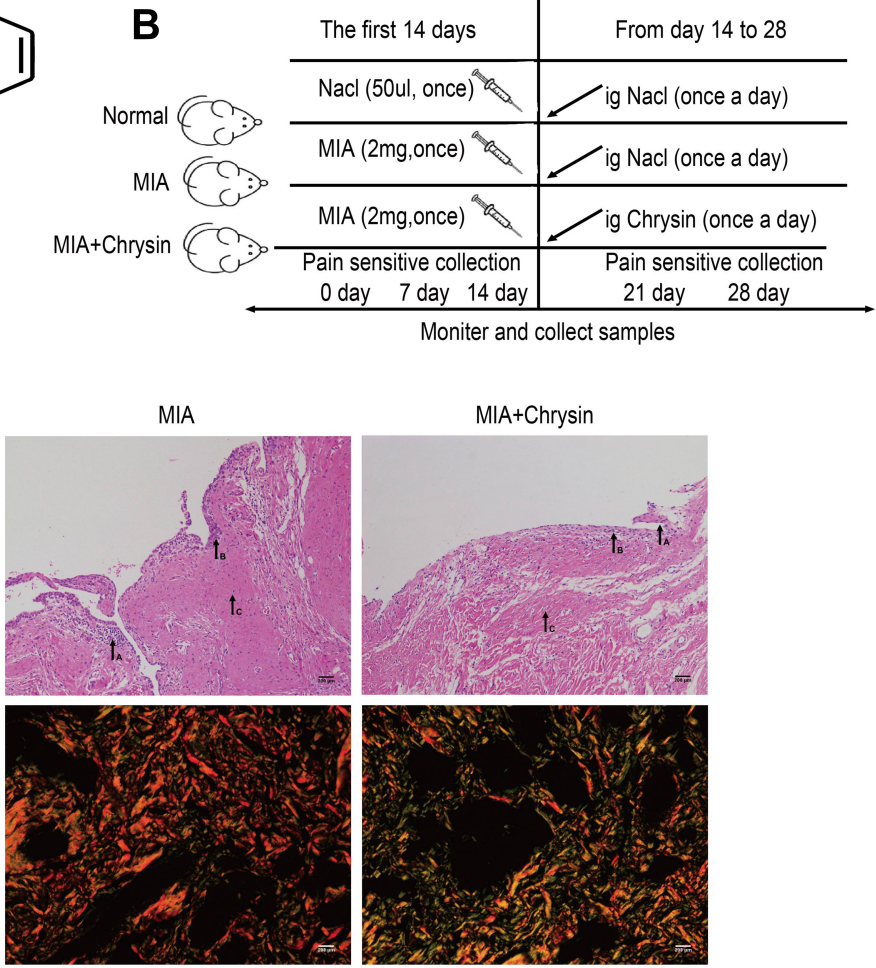

E

G

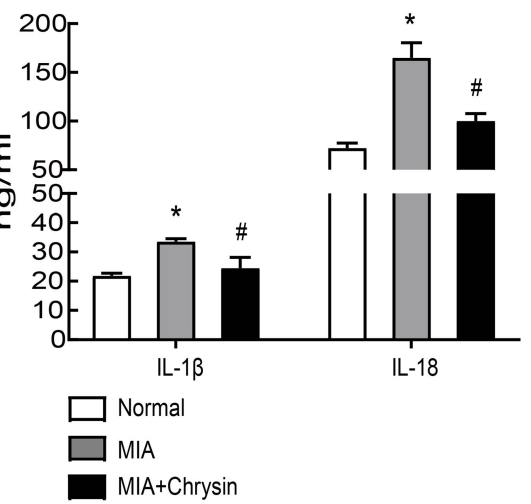

Figure I Chrysin reduced MIA-induced synovitis. (A) The chemical structural formula of Chrysin. (B) Pattern diagram of the animal experimental grouping. (C) H\&E (I00×, scale bar $=200 \mu \mathrm{m})$ and Sirius Red staining $(400 x$, scale bar $=200 \mu \mathrm{m})$ were applied to assess histopathological changes in the synovial membrane. (D) and (E) MIA-induced inflammation-related proteins were detected by WB. (F) IL-I $\beta$ and IL-I8 mRNA expression in synovial tissue was detected by qRT-PCR. (G) ELISA was used to detect rat serum inflammatory factors. The statistical data of the three independent experiments are expressed as the mean \pm SD, and significant differences among the groups are shown as $* \mathrm{P}<0.05$ vs the Normal group and ${ }^{\#} \mathrm{P}<0.05$ vs the MIA group. 

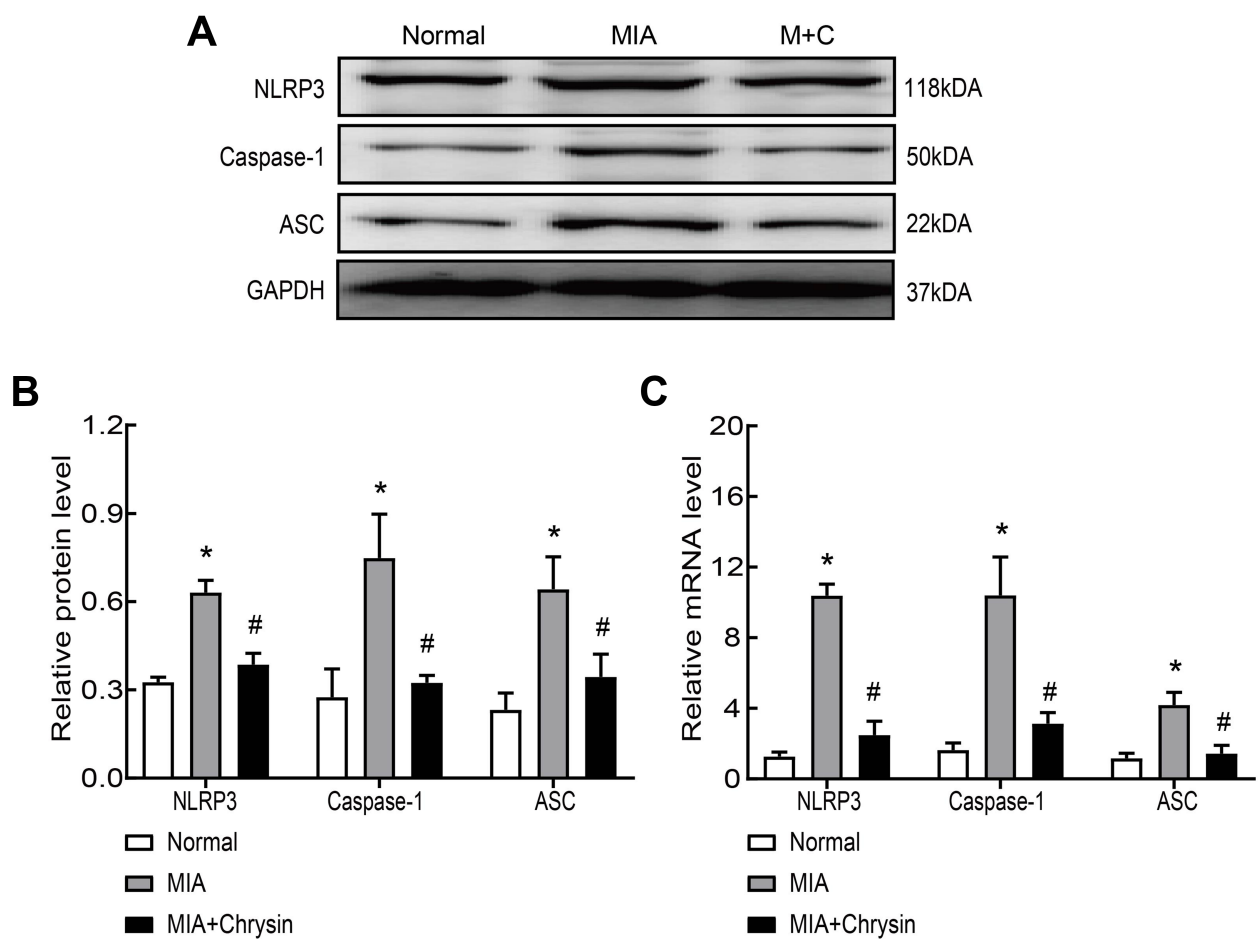

Figure 2 Chrysin suppressed MIA-induced NLRP3 inflammasome activation in vivo. (A and B) Western blot was performed to detect NLRP3, caspasel and ASC protein expression in the groups. Chrysin exerted an inhibitory effect. (C) QRT-PCR was performed to detect NLRP3, caspase I and ASC mRNA expression in the groups. Chrysin exerted an inhibitory effect. Statistical data of the three independent experiments are expressed as the mean \pm SD, and significant differences among the groups are shown as * $\mathrm{P}<0.05$ vs the Normal group and ${ }^{\#} \mathrm{P}<0.05$ vs the MIA group.

factors IL-1 $\beta$ and IL-18 was reduced in the cell supernatant upon treatment with Chrysin (Figure 4E).

\section{Chrysin Suppressed NLRP3 Inflammasome Activation in vitro}

Finally, we further explored the basic anti-inflammatory mechanisms of Chrysin at the cellular level. Western blot (Figure 5AB) and qRT-PCR (Figure 5C) were used to investigate the effect of Chrysin on LPS-induced NLRP3 activation. As shown in the images, compared with the normal group, LPS promoted NLRP3, caspase-1 and ASC mRNA expression; however, Chrysin suppressed this effect. Moreover, protein expression was consistent with qRTPCR results. Caspase-1 promoted NLRP3 inflammasome activation, which subsequently triggered downstream gene changes. Therefore, we further detected caspase-1 enzyme activity in each group. We found that LPS promoted the increase of caspase-1, whereas Chrysin inhibited this upregulation (Figure 5D).

\section{Discussion}

Osteoarthritis is highly prevalent in the United States and globally. It is the main cause of disability and it can negatively affect people's physical and mental health. ${ }^{1}$ Osteoarthritis is a disease of the whole joint that involves structural changes in articular cartilage, subchondral bone, ligaments, joint capsules, synovium, and muscles around the joints. ${ }^{2}$ These effects are directly related to clinical symptoms, such as joint swelling, synovitis, and inflammatory pain. Osteoarthritis is a complex pathological process involving multiple pathological pathways, but its etiology is unknown. Synovitis is one of the driving factors of the pathogenesis of KOA, which is derived from inflammatory cells infiltration of the lubricating membrane followed by synovial hyperplasia. The proliferating synovial membrane then produces a large number of proinflammatory cytokines, chemokines and proteases, which invade and destroy cartilage tissue. It is particularly worth mentioning that FLSs are central effectors in the development of synovitis. ${ }^{28}$ Steroids and nonsteroidal anti-inflammatory drugs are widely used in the anti-inflammatory and analgesic treatment of KOA. However, the side effects of these drugs are obvious. In recent years, an increasing number of natural compounds have gradually attracted attention due to their obvious anti-inflammatory effects and reduced side effects. Chrysin is an active ingredient extracted from the herb Scutellariae Radix that has various therapeutic effects, including immunomodulatory, anti-inflammatory, anti-oxidative 
A

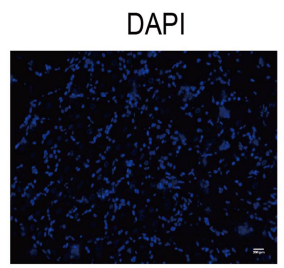

MIA
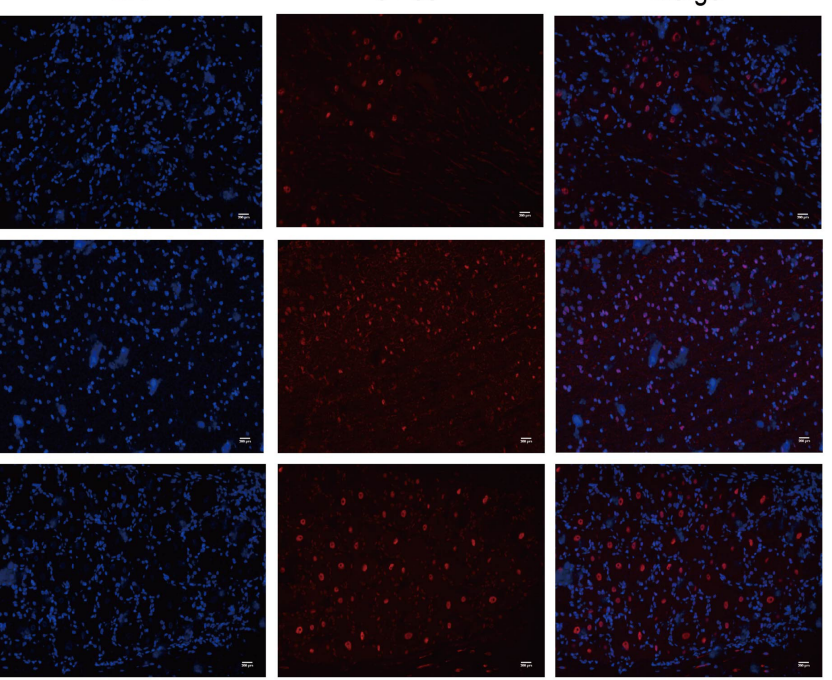

B

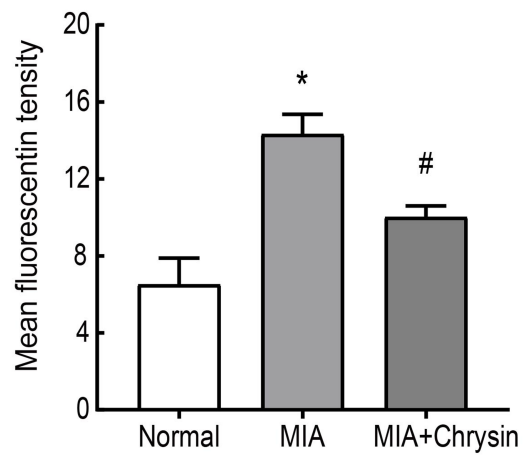

D

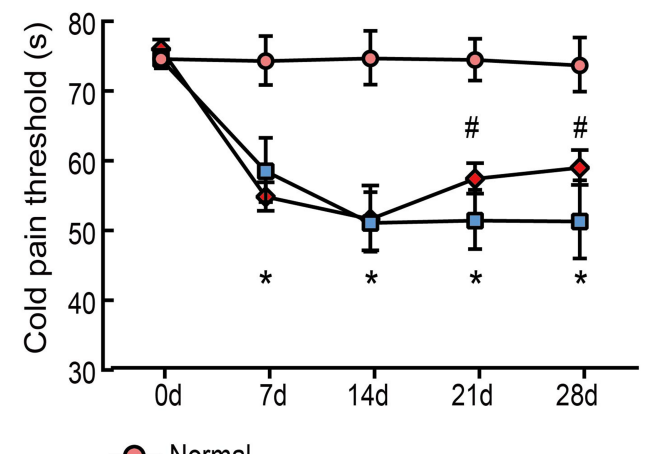

-O- Normal

$\neg$ MIA

MIA+Chrysin

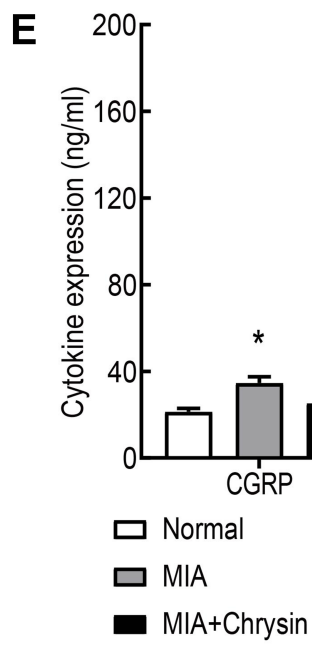

Figure 3 Chrysin Alleviated Pain and Increased the Cold Pain Threshold (s) and PWT (g) in Vivo. (A) and (B) c-Fos immunofluorescence was increased in the DRG of MIAtreated rats, compared to the Normal group, 200x, Scale bar $=200 \mu \mathrm{m}$. (C) and (D) PWT and cold pain threshold of each group. (E) CGRP and SP levels in rat serum were detected by ELISA. The statistical data of the three independent experiments are expressed as the mean \pm SD, and significant differences among the groups are shown as $* \mathrm{P}<0.05$ vs the Normal group and ${ }^{\#} \mathrm{P}<0.05$ vs the MIA group. 
A

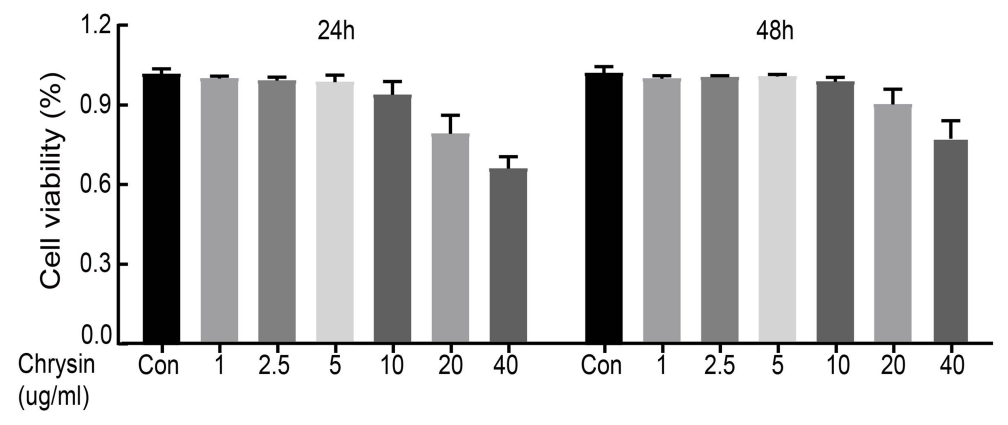

B

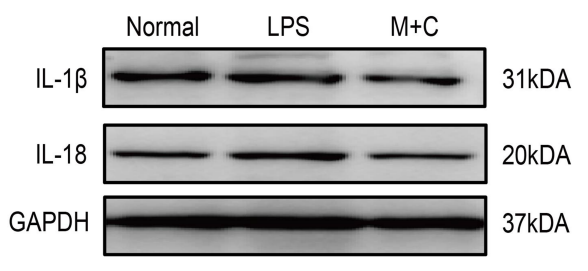

D

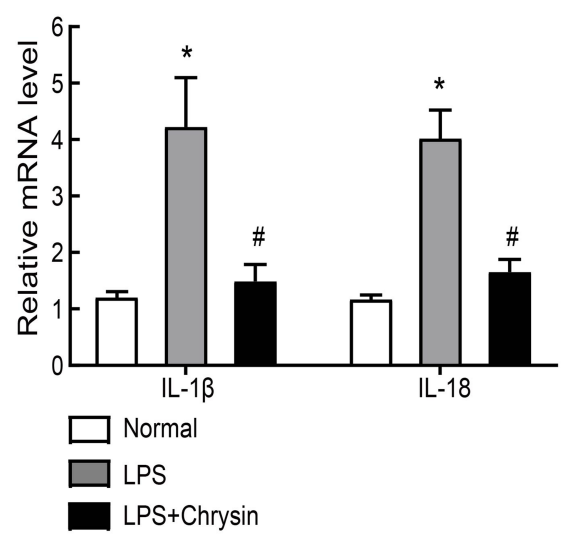

C

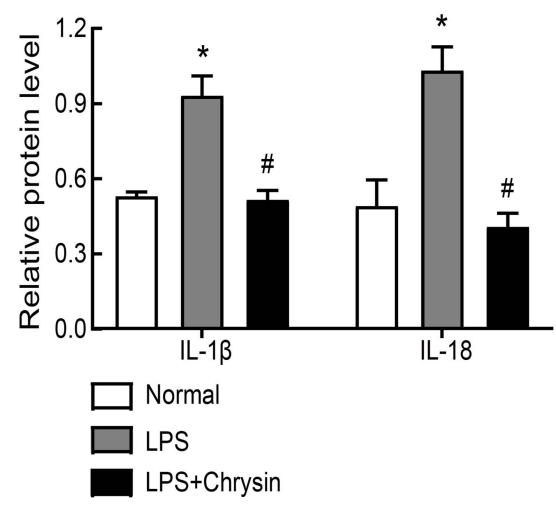

E

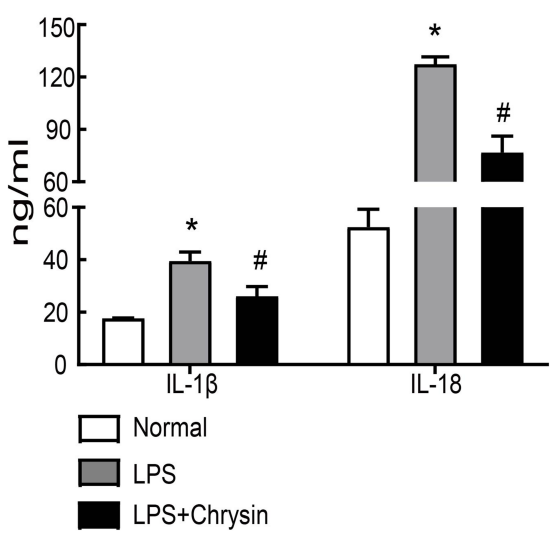

Figure 4 Chrysin reduced inflammatory factors of LPS-induced FLSs in vitro. (A) The CCK-8 assay was performed to investigate the cytotoxicity of different concentrations $(\mathrm{I}, 2,2.5,5,10,20$, and $40 \mathrm{ug} / \mathrm{mL})$ of Chrysin on FLSs. (B) and (C) Inflammation-related proteins and quantification analysis: IL-I $\beta$ and IL-I8 were determined by Western blot. (D) Genes of LPS-induced inflammatory factors were suppressed by Chrysin treatment. QRT-PCR was applied to evaluate the expression of inflammation-related genes. (E) Levels of inflammatory factor (IL-I $\beta$ and IL-18) in the FLSs supernatant were detected by ELISA. Statistical data of the three independent experiments are expressed as the mean $\pm \mathrm{SD}$, and significant differences among the groups are shown as $* \mathrm{P}<0.05$ vs the Normal group and ${ }^{\#} \mathrm{P}<0.05$ vs the LPS group.

stress and neuroprotective effects. Research in recent years has demonstrated that Chrysin has a wide range of anti-inflammatory effects. Chrysin improves allergic inflammation; ${ }^{29}$ inhibits ocular inflammation; ${ }^{16}$ reduces arthritis, ${ }^{17,18}$ specific dermatitis, ${ }^{30}$ colitis, ${ }^{31}$ and LPS-induced inflammation; ${ }^{32,33}$ and reduces kidney, ${ }^{34}$ myocardium, ${ }^{35}$ and brain damage ${ }^{36}$ caused by inflammation. Therefore, Chrysin, as a widely used anti-inflammatory drug, has the potential to reduce osteoarthritis. The active ingredient Chrysin is extracted from plants, so the future application of chrysin may serve as an inexpensive drug with increased efficacy and reduced side effects. Although studies have shown that Chrysin inhibits the development of human osteoarthritis through the NF- $\kappa$ B pathway and reduces the expression of IL-1 $\beta$-induced inflammatory mediators, ${ }^{17}$ this is the first study of the anti-inflammatory mechanism of Chrysin on the synovium and NLRP3 inflammasome. Our research shows that Chrysin not only has a positive anti-inflammatory effect but also can reduce inflammatory pain, and has the potential to be developed as a novel OA drug. 
A

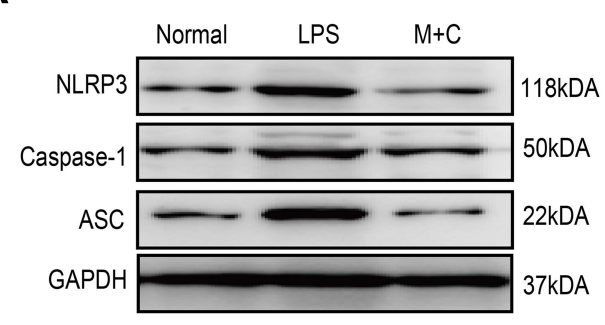

C

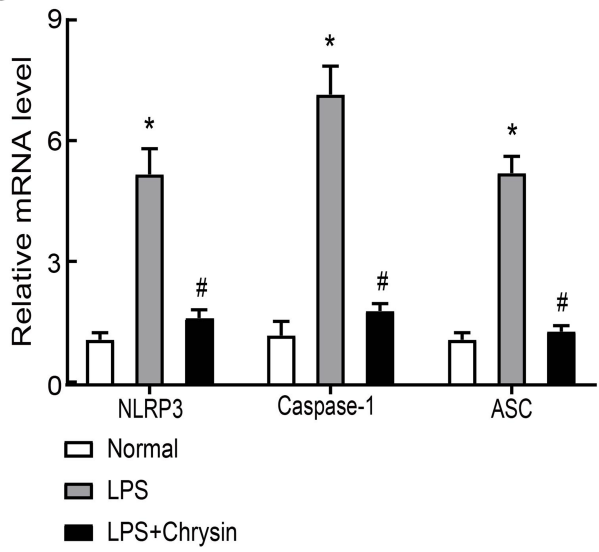

B

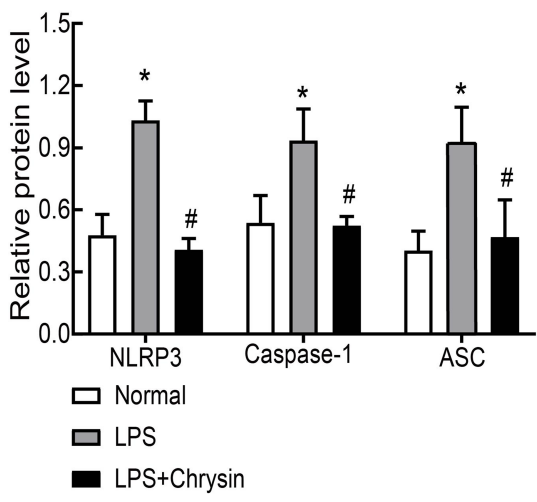

D

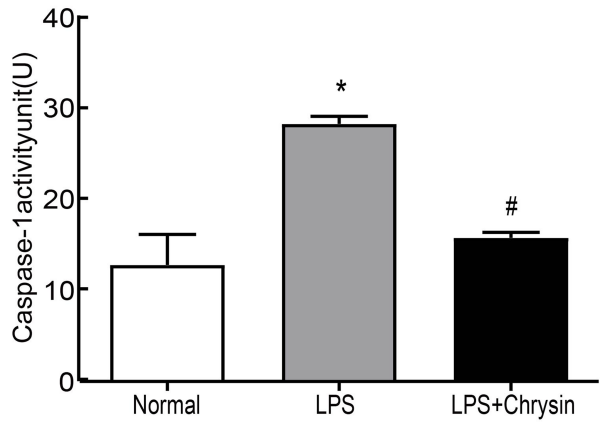

Figure 5 Chrysin suppressed NLRP3 inflammasome activation in vitro. Secreted protein (A and B) or mRNA (C) levels of NLRP3, caspasel and ASC in FLSs. (D) Caspase-I activity detection. The statistical data of the three independent experiments are expressed as the mean \pm SD, and significant differences among the groups are shown as $* \mathrm{P}<0.05$ vs the Normal group and ${ }^{\#} \mathrm{P}<0.05$ vs the LPS group.

In the past decade, the role of the NLRP3 inflammasome in inflammation has attracted attention, and numerous studies have been performed on uncontrolled infection, autoimmune diseases, neurodegenerative diseases, various autoimmune and autoinflammatory diseases, including metabolic disorders. ${ }^{37}$ The NLRP3 inflammasome is closely related to the pathogenesis of KOA and is involved in the cartilage destruction and synovitis of KOA. ${ }^{9}$ Two signaling models of NLRP3 activation are known, including the activation signal at the transcription level of the first step and the activation signal of the second step. Ultimately, NLRP3, ASC, and caspase 1 combine to form the inflammasome. Pro-caspase-1 is converted to caspase-1, which converts pro-IL-1 $\beta$ and pro-IL-18 into mature active forms (IL-1 $\beta$ and IL-18). ${ }^{8}$ It has been found in the research of joint diseases that caspase- 1 is one of the risk factors for osteoarthritis and accelerated cartilage destruction. ${ }^{38}$

In this work, our team sought to investigate the antiinflammatory mechanism of Chrysin in vitro and in vivo by targeting the NLRP3 inflammasome. First, CCK-8 was performed to investigate the effect of Chrysin at different concentrations on the cytotoxicity of FLS, and $5 \mathrm{ug} / \mathrm{mL}$ Chrysin exhibited no cytotoxicity. Next, compared with the normal group, mRNA and protein expression levels of caspase1, NLRP3 and ASC in KOA synovial tissues and FLSs were significantly upregulated; however, Chrysin reversed this trend. Besides, the KOA+Chrysin group exhibited downregulation of Caspase-1 activity compared with the KOA group. The evidence above suggests that Chrysin inhibited NLRP3 inflammasome activation in the synovial membrane of KOA.

Researchers have discovered that in the early stage of KOA, targeting synovitis can delay or prevent knee cartilage damage and osteophyte formation. Synovial fibroblasts in synovial tissues are effector cells of synovitis and are specific resident cells in body tissues and organs. Their main function is to maintain extracellular matrix (ECM) during wound healing and promote recovery and health. ${ }^{28}$ IL-1 $\beta$ and IL-18 are two key inflammatory factors that are considered to be involved in the pathogenesis of KOA. Anti-inflammatory studies on IL-1 $\beta$ are extensive, and it can independently induce inflammatory 
responses and catabolic effects that are related to a variety of signaling mechanisms. For example, IL-1 $\beta$ blocks the synthesis of ECM by chondrocytes and interferes with the synthesis of type II collagen and proteoglycans. ${ }^{28}$ IL-18 directly affects synovial cells and chondrocytes of the joints and increases the production of a series of cytokines and enzymes, such as PGE2 and IL-6, through autocrine pathways. ${ }^{39}$ In this paper, we reported that the administration of Chrysin effectively reduces IL- $1 \beta$ and IL-18 levels in the rat serum or supernatant of FLSs. H\&E and Sirius Red staining demonstrated that the KOA + Chrysin group exhibits reduced synovial inflammation compared with the KOA group. This finding is consistent with previous literature. ${ }^{17}$

Pain sensations can be divided into acute and chronic pain, as well as peripheral and central pain, and different types of pain are attributed to different etiological mechanisms, including inflammation and neuropathological mechanisms. The PWT or cold pain threshold is often used to directly assess the surrounding hyperalgesia. Usually, experimental research traditionally focuses on the dorsal root ganglia (DRG) to explore the mechanisms of inflammation-related pain. The DRG is located in the lumbar spine 3, 4, 5 and can be divided into three different types. Among these types, the second type of small neurons with axon terminals contains substance P (SP) and calcitonin generelated peptide (CGRP), which are two neuropeptides related to pain. As we all know, CGRP is highly related to hyperalgesia, and high levels of CGRP are often considered to be related to inflammation-related pain. ${ }^{40}$ In this paper, the reason why we chose the MIA model is that the MIA model is particularly relevant to OA-related pain, accompanied by hyperalgesia and allodynia in the major and minor parts of the rat, which has the advantages of simple induction, noninvasiveness, induce inflammation, easy formation of joint pain, and similar to pathology and pain changes in human osteoarthritis. In addition, the KOA+Chrysin group showed an increase in the cold pain threshold and PWT compared with the Normal group. Finally, we observed a decrease in serum CGRP and SP in KOA rats. Moreover, the c-Fos expression has become an important research tool in studies the basis of nociceptive sensory nerves. In this paper, we performed c-Fos immunofluorescence staining on the dorsal root ganglia. The staining results revealed a correlation between glial cell activation and chronic inflammatory pain.

Researches confirmed that the studies of NLRP3-mediated chronic aseptic inflammation are becoming increasingly popular, and evidence of NLRP3 activation has also been found in KOA. ${ }^{41}$ The downstream IL-1 $\beta$ and IL-18 have been highly correlated with OA pain. Our research have indicated that NLRP3 may be the effect target of Chrysin, which can effectively attenuate the NLRP3 inflammasome cascade, which is consistent with the various antiinflammatory effects of Chrysin confirmed by other scholars. In addition, Chrysin can significantly improve the pain behavior of KOA rats and reduce the expression of pain-related factors, indicating that Chrysin may relieve KOA synovitis through the NLRP3 pathway, and the reduction of inflammation leads to pain relief.

\section{Conclusions}

In conclusion, the findings of our study indicate that Chrysin may represent an effective agent to treat the LPS-induced FLS inflammatory environment in vitro and MIA-induced synovitis in vivo. Inhibition of NLRP3 inflammasome activation appears to be the basic mechanism of action of Chrysin. However, some limitations have been identified in the current study. First, it is not clear how Chrysin affects other important factors in the NLRP3 signaling pathway, such as pro-IL-1 $\beta$, pro-IL-18 and pro-caspase-1. Second, this study exclusively focused on the synovial membrane (without studying articular cartilage). We hope to address these limitations in future research.

\section{Abbreviations}

KOA, knee osteoarthritis; MIA, monoiodoacetic acid; FLSs, fibroblast-like synoviocytes; NLRP, nod-like receptor protein; ASC, apoptosis-associated speck-like protein containing a caspase-recruitment domain; IL-1 $\beta$, interleukin-1 $\beta$; IL-18, interleukin-18; SP, substance P; CGRP, calcitonin gene-related peptide; LPS, lipopolysaccharide; DRG, dorsal root ganglia; PWT, paw withdrawal threshold.

\section{Data Sharing Statement}

The data used to support the findings of this study are available from the corresponding author upon request.

\section{Funding}

The current work was supported by the National Natural Science Foundation of China (No. 81774334), the Leading Talents of Traditional Chinese Medicine Project (SLJ0207) and Nanjing University of Chinese Medicine "Jiangsu University Nursing Advantage Discipline Construction Project Funding Project" (2019YSHL085). 


\section{Disclosure}

The authors declare that they have no competing interests.

\section{References}

1. Vina ER, Kwoh CK. Epidemiology of osteoarthritis: literature update. Curr Opin Rheumatol. 2018;30(2):160-167. doi:doi:10. 1097/BOR.0000000000000479

2. Hunter DJ, Bierma-Zeinstra S. Osteoarthritis. Lancet. 2019;393 (10182):1745-1759. doi:doi:10.1016/S0140-6736(19)30417-9

3. Ishidou Y, Matsuyama K, Sakuma D, et al. Osteoarthritis of the hip joint in elderly patients is most commonly atrophic, with low parameters of acetabular dysplasia and possible involvement of osteoporosis. Arch Osteoporos. 2017;12(1):30. doi:doi:10.1007/ s11657-017-0325-4

4. Wallace G, Cro S, Dore C, et al. Associations between clinical evidence of inflammation and synovitis in symptomatic knee osteoarthritis: a cross-sectional substudy. Arthritis Care Res. 2017;69 (9):1340-1348. doi:doi:10.1002/acr.23162

5. Atukorala I, Kwoh CK, Guermazi A, et al. Synovitis in knee osteoarthritis: a precursor of disease? Ann Rheum Dis. 2016;75(2):390-395. doi:doi:10.1136/annrheumdis-2014-205894

6. Ostojic M, Ostojic M, Prlic J, Soljic V. Correlation of anxiety and chronic pain to grade of synovitis in patients with knee osteoarthritis. Psychiatr Danub. 2019;31(Suppl 1):126-130.

7. Ashraf S, Mapp PI, Shahtaheri SM, Walsh DA. Effects of carrageenan induced synovitis on joint damage and pain in a rat model of knee osteoarthritis. Osteoarthritis Cartilage. 2018;26(10):1369-1378. doi: doi:10.1016/j.joca.2018.07.001

8. McAllister MJ, Chemaly M, Eakin AJ, Gibson DS, McGilligan VE. NLRP3 as a potentially novel biomarker for the management of osteoarthritis. Osteoarthritis Cartilage. 2018;26(5):612-619. doi: doi:10.1016/j.joca.2018.02.901

9. Zhao LR, Xing RL, Wang PM, et al. NLRP1 and NLRP3 inflammasomes mediate LPS/ATP-induced pyroptosis in knee osteoarthritis. Mol Med Rep. 2018;17(4):5463-5469. doi:doi:10.3892/mmr.2018.8520

10. Jiang D, Chen S, Sun R, Zhang X, Wang D. The NLRP3 inflammasome: role in metabolic disorders and regulation by metabolic pathways. Cancer Lett. 2018;419:8-19. doi:doi:10.1016/j.canlet. 2018.01.034

11. Kelley N, Jeltema D, Duan Y, He Y. The NLRP3 inflammasome: an overview of mechanisms of activation and regulation. Int J Mol Sci. 2019;20:13. doi:doi:10.3390/ijms20133328

12. He Y, Hara H, Nunez G. Mechanism and regulation of NLRP3 inflammasome activation. Trends Biochem Sci. 2016;41(12):1012-1021. doi: doi:10.1016/j.tibs.2016.09.002

13. Williams CA, Harborne JB, Newman M, Greenham J, Eagles J. Chrysin and other leaf exudate flavonoids in the genus Pelargonium. Phytochemistry. 1997;46(8):1349-1353. doi:doi:10.1016/s0031-9422 (97)00514-1

14. Mohos V, Fliszar-Nyul E, Schilli G, et al. Interaction of chrysin and its main conjugated metabolites Chrysin-7-Sulfate and Chrysin-7-Glucuronide with serum albumin. Int $J$ Mol Sci. 2018;19:12. doi:doi:10.3390/ijms19124073

15. Zhao Q, Zhang Y, Wang G, et al. A specialized flavone biosynthetic pathway has evolved in the medicinal plant, Scutellaria baicalensis. Sci Adv. 2016;2(4):e1501780. doi:doi:10.1126/sciadv.1501780

16. Meng X, Fang S, Zhang Z, et al. Preventive effect of chrysin on experimental autoimmune uveitis triggered by injection of human IRBP peptide 1-20 in mice. Cell Mol Immunol. 2017;14 (8):702-711. doi:doi:10.1038/cmi.2015.107

17. Zheng W, Tao Z, Cai L, et al. Chrysin attenuates IL-1beta-Induced expression of inflammatory mediators by suppressing NF-kappaB in human osteoarthritis chondrocytes. Inflammation. 2017;40 (4):1143-1154. doi:doi:10.1007/s10753-017-0558-9
18. Darwish HA, Arab HH, Abdelsalam RM. Chrysin alleviates testicular dysfunction in adjuvant arthritic rats via suppression of inflammation and apoptosis: comparison with celecoxib. Toxicol Appl Pharmacol. 2014;279(2):129-140. doi:doi:10.1016/j.taap.2014.05.018

19. El KI, Abdelsalam RM, Elbrairy AI, Attia AS. Chrysin attenuates global cerebral ischemic reperfusion injury via suppression of oxidative stress, inflammation and apoptosis. Biomed Pharmacother. 2019;112:108619. doi:doi:10.1016/j.biopha.2019.108619

20. George MY, Esmat A, Tadros MG, El-Demerdash E. In vivo cellular and molecular gastroprotective mechanisms of chrysin; Emphasis on oxidative stress, inflammation and angiogenesis. Eur J Pharmacol. 2018;818:486-498. doi:doi:10.1016/j.ejphar.2017.11.008

21. Goes A, Jesse CR, Antunes MS, et al. Protective role of chrysin on 6-hydroxydopamine-induced neurodegeneration a mouse model of Parkinson's disease: involvement of neuroinflammation and neurotrophins. Chem Biol Interact. 2018;279:111-120. doi:doi:10.1016/j. cbi.2017.10.019

22. Zhang L, Zhang L, Huang Z, et al. Increased HIF-1alpha in knee osteoarthritis aggravate synovial fibrosis via Fibroblast-Like synoviocyte pyroptosis. Oxid Med Cell Longev. 2019;2019:6326517. doi: doi:10.1155/2019/6326517

23. Li TF, Ma J, Han XW, et al. Chrysin ameliorates cerebral ischemia/ reperfusion (I/R) injury in rats by regulating the $\mathrm{PI} 3 \mathrm{~K} / \mathrm{Akt} / \mathrm{mTOR}$ pathway. Neurochem Int. 2019;129:104496. doi:doi:10.1016/j. neuint.2019.104496

24. Wu P, Huang Z, Shan J, et al. Interventional effects of the direct application of "Sanse powder" on knee osteoarthritis in rats as determined from lipidomics via UPLC-Q-Exactive Orbitrap MS. Chin Med. 2020;15:9. doi:doi:10.1186/s13020-020-0290-5

25. Jasmin L, Kohan L, Franssen M, Janni G, Goff JR. The cold plate as a test of nociceptive behaviors: description and application to the study of chronic neuropathic and inflammatory pain models. Pain. 1998;75(23):367-382. doi:doi:10.1016/s03043959(98)00017-7

26. Yin S, Wang P, Xing R, et al. Transient receptor potential ankyrin 1 (TRPA1) mediates lipopolysaccharide (LPS)-Induced inflammatory responses in primary human osteoarthritic Fibroblast-Like synoviocytes. Inflammation. 2018;41(2):700-709. doi:doi:10.1007/ s10753-017-0724-0

27. Minten M, Blom A, Snijders GF, et al. Exploring longitudinal associations of histologically assessed inflammation with symptoms and radiographic damage in knee osteoarthritis: combined results of three prospective cohort studies. Osteoarthritis Cartilage. 2019;27 (1):71-79. doi:doi:10.1016/j.joca.2018.10.014

28. Mathiessen A, Conaghan PG. Synovitis in osteoarthritis: current understanding with therapeutic implications. Arthritis Res Ther. 2017;19(1):18. doi:doi:10.1186/s13075-017-1229-9

29. Yao J, Jiang M, Zhang Y, Liu X, Du Q, Feng G. Chrysin alleviates allergic inflammation and airway remodeling in a murine model of chronic asthma. Int Immunopharmacol. 2016;32:24-31. doi: doi:10.1016/j.intimp.2016.01.005

30. Choi JK, Jang YH, Lee S, et al. Chrysin attenuates atopic dermatitis by suppressing inflammation of keratinocytes. Food Chem Toxicol. 2017;110:142-150. doi:doi:10.1016/j.fct.2017.10.025

31. Dou W, Zhang J, Zhang E, et al. Chrysin ameliorates chemically induced colitis in the mouse through modulation of a PXR/ NF-kappaB signaling pathway. J Pharmacol Exp Ther. 2013;345 (3):473-482. doi:doi:10.1124/jpet.112.201863

32. Ha SK, Moon E, Kim SY. Chrysin suppresses LPS-stimulated proinflammatory responses by blocking NF-kappaB and JNK activations in microglia cells. Neurosci Lett. 2010;485(3):143-147. doi:doi:10. 1016/j.neulet.2010.08.064

33. Qi SM, Li Q, Jiang Q, Qi ZL, Zhang Y. [Chrysin inhibits lipopolysaccharide-induced inflammatory responses of macrophages via JAK-STATs signaling pathway]. Nan Fang Yi Ke Da Xue Xue Bao. 2018;38(3):243-250. Chinese. 
34. $\mathrm{Xu} \mathrm{M}$, Shi H, Liu D. Chrysin protects against renal ischemia reperfusion induced tubular cell apoptosis and inflammation in mice. Exp Ther Med. 2019;17(3):2256-2262. doi:doi:10.3892/ etm.2019.7189

35. Rani N, Bharti S, Bhatia J, Nag TC, Ray R, Arya DS. Chrysin, a PPAR-gamma agonist improves myocardial injury in diabetic rats through inhibiting AGE-RAGE mediated oxidative stress and inflammation. Chem Biol Interact. 2016;250:59-67. doi:doi:10.1016/j.cbi.2016.03.015

36. Sarkaki A, Farbood Y, Mansouri S, et al. Chrysin prevents cognitive and hippocampal long-term potentiation deficits and inflammation in rat with cerebral hypoperfusion and reperfusion injury. Life Sci. 2019;226:202-209. doi:doi:10.1016/j.lfs.2019.04.027

37. Su M, Wang W, Liu F, Li H. Recent progress on the discovery of NLRP3 inhibitors and their therapeutic potential. Curr Med Chem. 2020;27. doi:10.2174/0929867327666200123093544.
38. Wang X, Fan J, Ding X, Sun Y, Cui Z, Liu W. Tanshinone i inhibits IL-1beta-Induced apoptosis, inflammation and extracellular matrix degradation in chondrocytes $\mathrm{CHON}-001$ cells and attenuates murine osteoarthritis. Drug Des Devel Ther. 2019;13:3559-3568. doi: doi:10.2147/DDDT.S216596

39. Wojdasiewicz P, Poniatowski LA, Szukiewicz D. The role of inflammatory and anti-inflammatory cytokines in the pathogenesis of osteoarthritis. Mediators Inflamm. 2014;2014:561459. doi:doi:10.1155/2014/561459

40. Yamaguchi T, Ochiai N, Sasaki Y, et al. Efficacy of hyaluronic acid or steroid injections for the treatment of a rat model of rotator cuff injury. J Orthop Res. 2015;33(12):1861-1867. doi:doi:10.1002/jor.22976

41. Zhang L, Xing R, Huang Z, et al. Inhibition of synovial macrophage pyroptosis alleviates synovitis and fibrosis in knee osteoarthritis. Mediators Inflamm. 2019;2019:2165918. doi:doi:10.1155/2019/2165918

\section{Publish your work in this journal}

Drug Design, Development and Therapy is an international, peerreviewed open-access journal that spans the spectrum of drug design and development through to clinical applications. Clinical outcomes, patient safety, and programs for the development and effective, safe, and sustained use of medicines are a feature of the journal, which has also been accepted for indexing on PubMed Central. The manuscript management system is completely online and includes a very quick and fair peer-review system, which is all easy to use. Visit http://www. dovepress.com/testimonials.php to read real quotes from published authors. 